\title{
Abandono escolar y desvinculación de la escuela: perspectiva del alumnado
}

High School Dropout: The Students' Perspective

Abandon scolaire et détachement de l'école: la perspective des élèves Abandono escolar e desvinculação da escola: perspectiva do corpo discente

Fecha de recepción: 7 DE JUNIO DE 2013/ fecha de aceptación: 5 DE OCTUBRE DE 2013

Encuentre este artículo en http://magisinvestigacioneducacion.javeriana.edu.co/ doi:10.11144/Javeriana.M6-13.AEDE

Escrito por Francesca Salvà-MUT UnIVERSIDAD de LAS IsLAS Baleares Palma de Mallorca, España f.salva@uib.es

Miquel F. Oliver-Trobat UNIVERSIDAD DE LAS ISLAS BALEARES Palma de Mallorca, España m.oliver@uib.es

Rubén Comas-Forgas

\section{Resumen}

Este trabajo se centra en la problemática del abandono escolar temprano en España. El objetivo es profundizar en el conocimiento de las razones y procesos que conducen al mismo, a partir de la visión de los propios jóvenes. La metodología es cualitativa y se basa en el enfoque biográfico. Los resultados documentan la multiplicidad de factores que influyen en el abandono escolar temprano, así como su carácter de proceso. También reflejan los roles de los diversos actores y se destaca la influencia del grupo de iguales y las dificultades de los centros educativos y de las familias para cumplir su función educativa.

\section{Palabras clave autor}

Abandono escolar temprano, deserción escolar, escuela secundaria para adultos, enfoque biográfico.

\section{Palabras clave descriptor}

Deserción escolar, deserción en educación de adultos-España.
UNIVERSIDAD dE LAS IsLAS BALEARES

Palma de Mallorca, España

rubencomas@uib.es

Para citar este artículo / To cite this article / Pour citer cet article / Para citar este artigo /

Salvà-Mut, F., Oliver-Trobat, M.F., \& Comas-Forgas, R. (2014). Abandono escolar y desvinculación de la escuela: perspectiva del alumnado. magis, Revista Internacional de Investigación en Educación, 6 (13), 129-142.

\section{Transferencia a la práctica}

Los resultados de la investigación muestran la necesidad de prevenir el abandono escolar temprano y de combatir los procesos de desvinculación de la escuela que conducen al abandono de la misma. Ello implica actuar en el ámbito académico y en el de la vinculación social con la escuela desde los inicios de la escolarización. Hay que añadir la necesidad de actuaciones específicas para facilitar la transición entre la educación primaria y la secundaria y el establecimiento de políticas que promuevan la compatibilidad entre la formación y el empleo. 


\section{Key words author}

High School Dropout, Secondary Education

for Adults, Biographical Approach.

\section{Key words plus}

Dropouts, Adult Education Dropouts-Spain

\section{Mots clés auteur}

Abandon scolaire tôt, désertion scolaire, école secondaire pour adultes, perspective biographique.

\section{Mots clés descripteur}

Abandon des études

échec scolaire-Espagne.

\section{Abstract}

This study addresses the problem of high school dropout in Spain. It aims to gain a more profound understanding of the reasons and processes that lead to students leaving school, based on the point of view of the teenagers themselves. A qualitative methodology and a biographical approach were chosen. The results reveal the multiple factors that play a role, and the fact that high school dropout should be seen as a process. They also show the roles of the different actors, the influence of a group of equals, and the challenges of educational institutions and families to fulfill their educative duties.

\section{Transference to practice}

The results of this study show the need to prevent high school dropout and to fight the processes of detachment from the school that lead to dropout. To do so means to act both in the academic sphere and in the social ties to the school from the beginning. Additionally, specific actions are needed to make the transition between primary and secondary education easier, as well as policies that promote compatibility between education and employment.

\section{Résumé}

Ce travail se concentré dans la problématique de l'abandon scolaire tôt en Espagne. L'objectif est celui d'approfondir dans la connaissance des raisons et processus qui conduisent à l'abandon, à partir du regard des propres jeunes. La méthodologie est qualitative et elle est fondée dans la perspective autobiographique. Les résultats documentent la multiplicité de facteurs qui influencent dans l'abandon scolaire tôt, ainsi que son caractère de processus. Aussi ces résultats montrent les rôles des divers acteurs et on souligne l'influence du groupe d'égales et les difficultés des centres éducatifs et des familles pour accomplir sa fonction éducative.

\section{Transfert à la pratique}

Les résultats de la recherche montrent le besoin de prévenir l'abandon scolaire tôt et de combattre les processus de détachement de l'école qui conduisent à son abandon. Cela implique agir dans le domaine académique et dans l'attachement social avec l'école depuis le début de la scolarité. En plus il faut ajouter le besoin d'actuations spécifiques pour faire plus facile la transition entre l'éducation primaire et secondaire et l'établissement de politiques qui contribuent à la compatibilité entre la formation et l'emploie.

\section{Palavras-chave autor} Abandono escolar, deserção escolar, escola secundária para adultos, enfoque biográfico.

Palavras-chave descritor Décrocheurs, desistências de educação de adultos-España.

\section{Resumo}

Este trabalho se centra na problemática do abandono escolar na Espanha. O objetivo é aprofundar o conhecimento das razões e processos que conduzem ao mesmo, a partir da visão dos próprios jovens. A metodologia é qualitativa e se baseia no enfoque biográfico. Os resultados documentam a multiplicidade de fatores que influem no abandono escolar, bem como seu caráter de processo. Também revelam os papéis dos diversos atores e se destaca a influência do grupo de iguais e as dificuldades dos centros educativos e das famílias para cumprir sua função educativa.

\section{Transferência à prática}

Os resultados da pesquisa mostram a necessidade de prevenir o abandono escolar e combater os processos de desvinculação da escola que conduzem ao abandono da mesma. Isso implica atuar no âmbito acadêmico e no da vinculação social com a escola desde os inícios da escolarização. É necessário também alertar sobre a necessidade de atuações específicas para facilitar a transição entre a educação primária e a secundária e o estabelecimento de políticas que promovam a compatibilidade entre a formação e o emprego. 


\section{Introducción}

La investigación sobre abandono escolar temprano o prematuro en España está estrechamente vinculada con las políticas de educación y formación de la Unión Europea (UE). La tasa de abandono escolar prematuro (AEP) operativiza uno de los cinco ámbitos de referencia para realizar el seguimiento de los objetivos de educación y formación en la UE¹. La estrategia educación y formación 2020 plantea como objetivo para el año 2020 que la "proporción de abandonos prematuros de la educación y la formación debería estar por debajo del 10\%" (Consejo de la Unión Europea, 2009 , p. 7). A pesar de la disminución progresiva de esta tasa en España y en Europa, la situación actual, dista todavía bastante de los objetivos planteados por la UE: según los últimos datos disponibles referidos a 2011 la tasa española de AEP es de $26,5 \%$, mientras la media de la UE es de 13,5\% (Ministerio de Educación, Cultura y Deporte, 2013). Los datos son todavía más alarmantes en la Comunidad Autónoma de las Islas Baleares lugar donde se desarrolló el presente estudio con tasas de AEP superiores a un $30 \%$ (Tabla 1).

Tabla 1.

Evolución de la tasa de AEP en Europa, España y Baleares

\begin{tabular}{lllllllllllll}
\hline & \multicolumn{10}{c}{ Años } \\
\cline { 2 - 12 } & 2000 & 2001 & 2002 & 2003 & 2004 & 2005 & 2006 & 2007 & 2008 & 2009 & 2010 & 2011 \\
\hline Baleares & 42,0 & 40,7 & 40,3 & 41,4 & 42,5 & 39,3 & 36,5 & 43,1 & 43,2 & 40,8 & 36,7 & 30,7 \\
\hline España & 29,1 & 29,7 & 30,7 & 31,6 & 32,0 & 30,8 & 30,5 & 31,0 & 31,9 & 31,2 & 28,4 & 26,5 \\
\hline UE 27 & 17,6 & 17,2 & 17,0 & 16,5 & 16,0 & 15,8 & 15,5 & 15,1 & 14,9 & 14,4 & 14,1 & 13,5 \\
\hline \hline
\end{tabular}

Fuente: elaboración propia con datos del Ministerio de Educación, Cultura y Deporte (2013)

La revisión de la literatura muestra que la investigación realizada en España sobre el tema está basada en la explotación de datos de fuentes secundarias (Informes PISA, Encuesta de población activa, etc.); ejemplos de este tipo de aproximaciones son los aportes de Casquero y Navarro (2010), Fernández, Muñoz, Braña y Antón (2010) y García, Casal, Merino y Sánchez (2013). Este tipo de trabajos se centra sobre todo en ejercicios comparativos entre países y en la determinación de factores asociados con el fracaso escolar y el AEP. En España, existen algunas aportaciones empíricas notables, tal es el caso de los trabajos de Mora (2010) sobre los determinantes del abandono escolar y Adame y Salva (2010) acerca del abandono escolar y de la transición a la vida activa. Sin embargo, es escasa la investigación respecto a la interacción entre los diversos factores y los procesos de desvinculación de la escuela, aun como elementos centrales para la comprensión de las situaciones de AEP (Blaya, 2010; Dale, 2010;
Descripción del artículo | Article description | Description de l'article | Artigo descrição

Artículo de investigación en el que se estudia el abandono escolar temprano y la desvinculación de la escuela desde la perspectiva del alumnado. Se deriva del proyecto de investigación "Abandono escolar prematuro y retorno a la educación en Baleares: historias de vida del alumnado de la Educación Permanente de Personas Adultas".

1 Se establece como indicador de dicho abandono el porcentaje de población de 18 a 24 años que tiene el título de educación secundaria de primera etapa o inferior y que no sigue ningún tipo de enseñanza o formación. En el sistema educativo español, el título de educación secundaria inferior o de primera etapa corresponde al de Graduado/a en Educación Secundaria Obligatoria (GESO). De acuerdo con la Clasificación Internacional de Educación (CINE), se trata de personas con nivel CINE 2 (educación secundaria inferior o de primera etapa) o inferior. 
Rumberger, 2004). En esta línea, en nuestro país se destacan los estudios realizados por Fernández-Enguita, Mena y Riviere (2010).

El presente trabajo tiene como objetivo profundizar en el conocimiento de las causas y los procesos que conducen al AEP. Para ello, adoptamos una perspectiva metodológica basada en las historias de vida de un grupo de jóvenes que abandonó la escuela sin obtener el título de Graduado/a en Educación Secundaria Obligatoria (GESO) y que están cursando la Educación Secundaria para Personas Adultas (ESPA). El análisis de sus narraciones contribuye a profundizar en el conocimiento de las razones y de los procesos de abandono y aporta elementos de interés para la reflexión y la mejora educativa.

\section{Las causas del AEP: factores, interacciones y procesos}

La literatura internacional sobre las causas del AEP se fundamenta en el estudio de los factores de riesgo, las interacciones entre estos factores y los procesos que conducen al abandono y se señala la existencia de cuatro características básicas del mismo: la multidimensionalidad, el carácter de proceso, las dificultades para establecer relaciones causa-efecto, y las interrelaciones entre factores individuales y contextuales (Blaya, 2010; Dale, 2010; Rumberger, 2004).

\section{Los factores de riesgo y sus interacciones}

El estudio de los factores de riesgo persigue identificar los elementos vinculados al abandono temprano. Estos factores son múltiples y heterogéneos y se producen en tres ámbitos: microsocial, mesosocial y macrosocial. En la Tabla 2 se presenta una síntesis de los mismos.

Tabla 2.

Abandono escolar temprano. Factores de riesgo

\begin{tabular}{|c|c|c|}
\hline Nivel & Dimensiones & Factores \\
\hline \multirow[t]{2}{*}{ 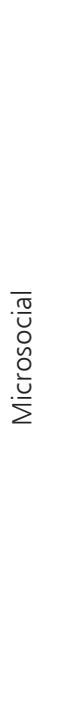 } & Características individuales & $\begin{array}{l}\text { - Generales con influencia en la escolarización e influenciadas por } \\
\text { la misma: escasas aspiraciones educativas; problemas de autoes- } \\
\text { tima, seguridad, habilidades sociales; problemas de salud física } \\
\text { y/o mental que afecten el comportamiento, lleven a ausentarse } \\
\text { y/o a un consumo inadecuado de medicamentos; problemas de } \\
\text { comportamiento internalizados (ansiedad, estado depresivo) o } \\
\text { externalizados (delincuencia, agresividad); adicciones; embarazo } \\
\text { adolescente. } \\
\text { - Relacionadas directamente con la escolarización: experiencia ne- } \\
\text { gativa de la escuela; falta de habilidades académicas, dificultades } \\
\text { de aprendizaje, retraso escolar, necesidades educativas especiales; } \\
\text { débil participación en la vida de la escuela y débil sentimiento de } \\
\text { pertenencia; malas relaciones con el profesorado; falta de mo- } \\
\text { tivación y desánimo; problemas de adaptación a las exigencias } \\
\text { escolares; comportamientos disruptivos; absentismo; numerosos } \\
\text { cambios de centro. }\end{array}$ \\
\hline & Relaciones interpersonales & $\begin{array}{l}\text { - Con los iguales: influencias negativas por parte de los pares; falta } \\
\text { de relaciones significativas. } \\
\text { - Con los adultos: falta de relaciones significativas con adultos que } \\
\text { puedan constituir un referente positivo. }\end{array}$ \\
\hline
\end{tabular}




\begin{tabular}{|c|c|c|}
\hline Nivel & Dimensiones & Factores \\
\hline \multirow{4}{*}{ 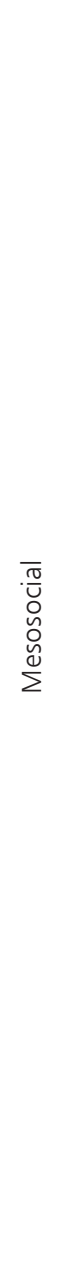 } & Familia & $\begin{array}{l}\text { - Dificultades familiares de tipo psicológico y socioeconómico. } \\
\text { - Débil cohesión familiar y dinámicas familiares difíciles. } \\
\text { - Insuficiente relación e implicación con los procesos de esco- } \\
\text { larización. } \\
\text { - Expectativas familiares de asunción temprana de roles adultos re- } \\
\text { lacionados con el empleo y/o con los cuidados. }\end{array}$ \\
\hline & Escuela & $\begin{array}{l}\text { - Clima de centro y/o de clase negativo. } \\
\text { - Orientación personal, escolar y profesional inadecuada. } \\
\text { - Actitudes negativas del profesorado hacia el alumnado. } \\
\text { - Ausencia o insuficiencia de relaciones significativas entre alumna- } \\
\text { do y profesorado y otras personas adultas de la escuela. } \\
\text { - Tamaño de la escuela: las escuelas pequeñas facilitan la relación } \\
\text { significativa entre alumnado y profesorado. } \\
\text { - Falta de supervisión y control en el aula. } \\
\text { - Elevado número de alumnado por aula. } \\
\text { - Falta de acompañamiento y valorización del alumnado. } \\
\text { - Políticas de admisión de la escuela. } \\
\text { - Políticas y prácticas para promover la "eficacia global" de la es- } \\
\text { cuela que llevan a suspensiones, expulsiones, cambios de escuela } \\
\text { forzados. } \\
\text { - Falta de oportunidades de formación para el profesorado. } \\
\text { - Falta de proyecto educativo de centro, falta de recursos educativos } \\
\text { y de apoyo de la dirección. } \\
\text { - Pedagogía inapropiada centrada en los contenidos del currículum } \\
\text { más que en el alumnado. }\end{array}$ \\
\hline & Grupo de iguales & $\begin{array}{l}\text { - Amistades con bajo nivel académico. } \\
\text { - Amistades con comportamientos poco adaptados a las exigen- } \\
\text { cias escolares. }\end{array}$ \\
\hline & Comunidad & $\begin{array}{l}\text { - Mercados de trabajo locales con amplia oferta de empleo con } \\
\text { bajos requerimientos educativos. } \\
\text { - Infravaloración de los títulos académicos y afirmación del trabajo } \\
\text { manual. } \\
\text { - Representaciones sociales tradicionales en relación con la partici- } \\
\text { pación de las mujeres en el empleo, que se subordina a las tareas } \\
\text { de cuidados familiares. }\end{array}$ \\
\hline \multirow{3}{*}{ 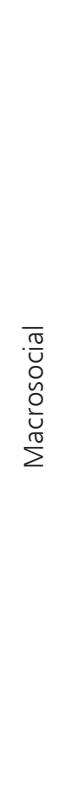 } & $\begin{array}{l}\text { Sistema de educación y } \\
\text { formación }\end{array}$ & $\begin{array}{l}\text { - Duración de la escolaridad obligatoria y características. } \\
\text { - Normativa relativa a la repetición de curso, ratios, sistemas de eva- } \\
\text { luación, admisión de alumnado, etc. } \\
\text { - Sistema de formación y acceso a la docencia y a la gestión y direc- } \\
\text { ción de centros educativos. } \\
\text { - Programas de prevención del abandono escolar. } \\
\text { - Gasto en educación y calidad y eficiencia del gasto. } \\
\text { - Programas de educación postobligatoria. } \\
\text { - Programas de educación y formación a lo largo de la vida. } \\
\text { - Programas de orientación y acompañamiento para el retorno a la } \\
\text { educación de jóvenes y adultos. }\end{array}$ \\
\hline & $\begin{array}{l}\text { Relaciones entre } \\
\text { formación y empleo }\end{array}$ & $\begin{array}{l}\text { - Legislación laboral y posibilidades de combinar formación y empleo. } \\
\text { - Características transición educación-empleo. } \\
\text { - Características del tejido productivo y de la oferta de empleo. } \\
\text { - Disponibilidad de formación de calidad. } \\
\text { - Conjunto de oportunidades y expectativas que ofrece el mercado } \\
\text { de trabajo. }\end{array}$ \\
\hline & $\begin{array}{l}\text { Valores sociales } \\
\text { dominantes }\end{array}$ & $\begin{array}{l}\text { - Sociedad que valora el consumo. } \\
\text { - Sociedad que vehicula el atractivo del éxito inmediato. } \\
\text { - Sociedad que valoriza el presente e ignora la proyección de futuro. } \\
\text { - Poca valoración social de la educación. } \\
\text { - Culturas juveniles alejadas de la cultura escolar. }\end{array}$ \\
\hline
\end{tabular}

Fuente: elaboración propia con datos de Blaya (2010); Consejo Económico y Social (2011); Dale (2010); Desmarais (2012); González (2006); Martínez y Álvarez (2005); Rumberger (2004); Salvà-Mut y SuredaNegre (2012) 
La identificación de los factores de riesgo permite deconstruir las causas del AEP, pero este ejercicio es insuficiente para entender el fenómeno en su globalidad. El valor predictivo de los factores de riesgo se produce a partir de la acumulación y de determinadas combinaciones de los mismos, por lo que en todos los casos se halló interacción entre factores personales, familiares y escolares (Blaya, 2010).

Para comprender las causas del AEP, conviene abordarlo desde la multidimensionalidad (acumulación de factores de riesgo e interacciones) y combinarlo con la dimensión temporal (el carácter de proceso). Las situaciones y percepciones que llevaron a dejar la escuela en un momento preciso tienen que analizarse como parte de un proceso, sin confundirse con las causas consideradas en su globalidad.

En el marco de la multidimensionalidad y temporalidad que caracterizan nuestro objeto de estudio, las principales aportaciones derivan de las investigaciones sobre la influencia de la familia, la escuela, los iguales y la comunidad, así como de los procesos de desvinculación o desenganche de la escuela.

Las características familiares, en especial su nivel socioeconómico, son reconocidas como un elemento que, por sí solo, contribuye en gran medida al éxito en la escuela. No obstante, como ya señaló Coleman (1988), el capital humano (educación parental) y financiero (ingresos parentales) son insuficientes para explicar la relación entre características familiares y éxito escolar. El capital social, que se manifiesta en las relaciones que los padres tienen con sus hijos, otras familias y la escuela, también tiene influencia en el éxito escolar, independientemente de los efectos del capital humano y económico. Dale (2010) señala como prácticas parentales más relacionadas con el abandono escolar temprano la falta de supervisión y un estilo parental permisivo, las bajas aspiraciones y la poca vinculación en relación con la escolarización de los hijos.

Las relaciones positivas entre alumnado y profesorado reducen la probabilidad de abandono, en particular entre estudiantes de alto riesgo. Este tipo de relación está ligada a diversas características de los centros educativos, entre las que se destaca el tamaño de los mismos: las escuelas pequeñas, las que tienen más probabilidades de facilitar la vinculación entre estudiantes y profesorado (Lee \& Burkam, 2003; Rumberger, 2004).

También se ha documentado la influencia de los grupos de iguales en el AEP. Tener amigos o hermanos que han abandonado incrementa la posibilidad de hacerlo, a la vez que se aumenta la posibilidad de éxito cuando amigos y hermanos lo han conseguido (Dale, 2010; Häfeli \& Schellenberg, 2009; Millet \& Thin, 2005; Morris \& Pullen, 2007; Rumberger, 2004).

Respecto a la comunidad, los mercados de trabajo locales con una amplia oferta de empleo de baja cualificación propician la salida temprana del sistema educativo (Consejo Económico y Social, 2009; Eckert, 2006; Lamamra \& Masdonati, 2009; Roksa \& Velez, 2010). El impacto de la facilidad para encontrar empleo en el abandono escolar temprano viene determinado, en gran parte, por los valores sociales dominantes, las características de los sistemas de educación y formación y las políticas educativas (Salvà-Mut \& Sureda-Negre, 2012).

\section{El proceso de abandono}

Para responder con mayor precisión a la pregunta sobre quiénes abandonan de manera prematura la escuela y por qué, hay que añadir una dimensión fundamental: la del proceso que lleva al abandono. Para entenderlo, debemos centrarnos en los propios estudiantes y en un concepto central: el de compromiso o vinculación con la escuela (engagement). Este compromiso es de dos tipos: académico y social. El primero guarda relación con los aprendizajes y los aspectos más formales de la escuela, mientras que el segundo hace referencia a los aspectos sociales e informales, entre los que sobresalen las relaciones con iguales y con adultos. El abandono escolar es considerado el estadio final de un proceso dinámico y acumulativo de desvinculación, alejamiento o retirada de la escuela (Morris \& Pullen, 2007; Rumberger, 2004).

Este proceso comienza a veces en los primeros años de escolaridad y en otros casos se inicia en la transición entre la educación primaria y la educación secundaria o durante la educación secundaria, en estrecha relación con los procesos de desarrollo propios de la adolescencia. En ambos casos, el período de transición entre la educación primaria y secundaria que se realiza entre los 11 y los 14 años es crítico en cuanto al proceso de desvinculación de la escuela (Feyfant, 2012; MenaMartínez, Fernández-Enguita \& Riviere-Gómez, 2010; Millet \& Thin, 2005; Morris \& Pullen, 2007).

Los estudios sobre los procesos de abandono en España abordan el abandono escolar como el resultado final de un proceso de "desenganche escolar", como "la lenta y progresiva acumulación de fuentes de alejamiento de la escala de valores, las pautas de actuación y los símbolos de identificación con la escuela" (Mena-Martínez, Fernández-Enguita \& Riviere-Gómez, 2010, p. 123) e identifican el paso a la educación secundaria como un período bastante crítico.

\section{Objetivos}

En este artículo se estudia el abandono escolar temprano y los procesos de desvinculación de la escuela, desde la perspectiva de la experiencia del propio alumnado y desde una visión multidimensional, 
perspectiva y visión que enfatizan las interacciones entre los factores a escala micro, meso y macrosocial y el carácter de proceso. Con ello se pretende contribuir al conocimiento de los principales factores y las interacciones que conducen al abandono escolar temprano en España y describir las características de los procesos de desvinculación de la escuela.

El objetivo concreto es contribuir a dar respuesta a las siguientes preguntas de investigación:

- ¿Cuáles son las principales interacciones que se producen entre los diversos factores que conducen al AEP?

- ¿Qué características presentan los procesos de desvinculación de la escuela?

- ¿Cuáles son los diversos momentos o las etapas y las situaciones críticas en el proceso de desvinculación?

- ¿ ¿Qué papel desempeñan los diversos actores (alumnado, profesorado, familia, comunidad)?

\section{Metodología}

La perspectiva biográfica es el referente metodológico adoptado en este trabajo. Es un enfoque de la corriente cualitativa o interpretativa que tiene como elemento central las historias de vida, que se construyen a partir de la entrevista biográfica o narrativa. Según Bertaux (1997), la historia de vida "resulta de una forma particular de entrevista, la entrevista narrativa, durante la cual un investigador [...] pide a la persona que denominamos "sujeto" que le cuente toda o una parte de la experiencia vivida" (p. 6).

El uso de las historias de vida en Ciencias Sociales y Humanas puede realizarse desde diversos enfoques (Bertaux, 1997; Desmarais, 2009; Ferraroti, 2011; Hernández, Sancho \& Rivas, 2011; Johnson, 2007; Pujadas, 1992; Rivas, Hernández, Sancho \& Núñez, 2012). En esta investigación asumimos la perspectiva etnosociológica (Bertaux, 1997), cuya finalidad es estudiar "un fragmento particular de la realidad histórico-social, un objeto social; comprender cómo funciona y cómo se transforma, poniendo el acento sobre las configuraciones de las relaciones sociales, los mecanismos, los procesos, las lógicas de acción que los caracterizan" (p. 7).

En este contexto, nuestro objeto de estudio es lo que Bertaux denomina una categoría de situación. Lo que es común es la "situación particular", situación que "es social, en la medida en que engendra obligaciones y lógicas de acción que tienen aspectos comunes, en que es percibida a través de esquemas colectivos y en que eventualmente es tratada por una misma institución". Las historias de vida son pertinentes porque permiten captar "los mecanismos y procesos por los cuales los sujetos se encuentran en una situación determinada, y cómo se esfuerzan en gestionar esta situación" (Bertaux, 1997, p. 15).

Se pretende un acercamiento a la realidad para comprender el funcionamiento de una parte de la misma. Para ello, se elige como informantes a determinadas personas (en nuestro caso personas que comparten una situación particular) que aportan una visión distinta a la de otros actores sociales.

\section{Participantes}

La población objeto de estudio está formada por treinta jóvenes (trece mujeres y diecisiete hombres) de 16 a 20 años (media de edad de 17,9 años), que abandonaron la escuela sin obtener la titulación de Graduado/da 
en Educación Secundaria Obligatoria (GESO) y que, en el momento de la investigación, cursaban estos estudios en la modalidad de Educación Secundaria para Personas Adultas (ESPA) en Baleares (España).

La mayoría de los y las jóvenes viven con su familia de origen y solo cuatro mujeres están emancipadas. De ellas, una vive con su pareja, una vive sola en una vivienda cedida por la familia de origen y dos en un piso compartido. Ninguna de las personas entrevistadas tiene hijos.

Los centros fueron seleccionados de forma que representaran la heterogeneidad territorial de las Islas Baleares. El resultado fue la selección de siete de los catorce centros que imparten ESPA en Baleares: uno de Ibiza (el único de la isla); uno de Menorca (hay dos); cinco de Mallorca (de un total de once). De los cinco de Mallorca, tres están en un entorno urbano (la ciudad de Palma de Mallorca), uno está en un municipio de la costa y otro en un pueblo del interior.

Se informó a los centros que la participación del alumnado en las entrevistas debía ser voluntaria y se les pidió que proporcionaran un listado con los datos necesarios para contactar las personas interesadas en participar en el estudio. En la selección de las personas que se habían ofrecido como voluntarias se tuvieron en cuenta la edad y el sexo para garantizar una representación equilibrada.

\section{Procedimiento}

Las entrevistas se realizaron entre los meses de febrero y abril de 2008, por parte de tres entrevistadoras instruidas para la tarea. La duración de las mismas ha oscilado entre 45 minutos y 2 horas. En la Tabla 3 se señalan las dimensiones tratadas en la entrevista que se fundamentan en los trabajos de Merete (2006), Rioux (1998), Simard (1997), Smale (2001) y Vaillancourt (1998).

Tabla 3.

Entrevista biográfica. Categorías y subcategorías tratadas en las entrevistas

\begin{tabular}{|c|c|}
\hline Categorías & Subcategorías \\
\hline Situación actual & $\begin{array}{l}\text { - Estudios en curso y motivaciones } \\
\text { - Trabajo } \\
\text { - Ocio } \\
\text { - Usos del tiempo } \\
\text { - Emancipación } \\
\text { - Familia } \\
\text { - Amistades } \\
\text { - Pareja }\end{array}$ \\
\hline Abandono escolar antes de obtener el título de GESO & $\begin{array}{l}\text { - Razones del abandono } \\
\text { - Sentimientos asociados con el abandono } \\
\text { - Proceso de abandono } \\
\text { - Biografía escolar } \\
\text { - Sentimientos en relación con la escuela } \\
\text { - Familia } \\
\text { - Amistades } \\
\text { - Pareja } \\
\text { - Profesorado }\end{array}$ \\
\hline Retorno/s a la educación y a la formación y perspectivas de futuro & $\begin{array}{l}\text { - Actividades y usos del tiempo después de dejar los es- } \\
\text { tudios de ESO } \\
\text { - Razones del retorno a la educación } \\
\text { - Valoración de las actividades de educación y formación } \\
\text { - Expectativas personales, académicas y profesionales }\end{array}$ \\
\hline
\end{tabular}

Fuente: elaboración propia con datos de Merete (2006); Rioux (1998); Simard (1997); Smale (2001); Vaillancourt (1998) 
Las entrevistas fueron grabadas y se transcribió el contenido literal de las mismas. También se diligenció una plantilla de observaciones que resumía las principales características del lugar donde se había realizado la entrevista, así como los aspectos más significativos del comportamiento de la persona entrevistada.

Una vez transcritas, se procedió al análisis de contenido. En un primer momento se revisó cada historia y se deconstruyó en unidades de sentido o temas, surgidos de las entrevistas. En una segunda fase, de acuerdo con el marco teórico de referencia, se agruparon los temas en categorías y subcategorías. Es un tipo de análisis según una lógica inductiva moderada, caracterizada por una posición intermedia entre lo que Savoie-Zajc (2011) denomina una lógica típicamente inductiva, en la que el marco teórico es retomado solo al final de la investigación y una lógica inductiva deliberativa, en la que el marco teórico guía todo el proceso de análisis.

Con el objetivo de controlar los principios de validez del estudio, se adoptó una estrategia de triangulación a la hora del análisis de contenido de todas las historias de vida. Este ejercicio se basó en: a) consensuar entre los tres investigadores participantes, en la fase de análisis de datos, las unidades de sentido de los discursos de los participantes; b) consensuar las categorías y subcategorías en que se englobaban las diferentes unidades de sentido; c) analizar cada entrevista por parte de cada investigador (triple análisis de contenido); d) consensuar los resultados de los diversos análisis de cada entrevista generando una versión única para cada caso.

Se procedió a la codificación de los datos con la ayuda del programa informático NUD IST para Windows. Finalizada esta fase, se sistematizaron y depuraron los resultados y se agruparon por categorías y subcategorías. A partir del análisis de los datos de cada categoría se elaboró y redactó el informe de resultados globales de la investigación.

\section{Resultados}

Los resultados de nuestro trabajo se presentan agrupados en cuatro apartados: dos centrados en los factores que llevan al abandono escolar y dos relativos al proceso de desvinculación con la escuela subyacente al abandono.

Los malos resultados académicos aparecen como principal desencadenante de un abandono provocado por múltiples causas

El examen de las narraciones de los y las jóvenes participantes en el estudio documenta el hecho de que los malos resultados académicos son el principal desencadenante del abandono escolar antes de obtener el título de GESO. De las treinta personas entrevistadas, solo dos obtenían buenos resultados académicos y tuvieron que dejar los estudios como consecuencia de situaciones personales traumáticas (problemas de salud y dificultades económicas). En los otros veintiocho casos, el motivo fue el bajo rendimiento académico, manifestado en repeticiones de curso y suspensiones.

En la mayoría de casos son los y las jóvenes quienes deciden abandonar. Las principales razones que esgrimen son las siguientes:

a) Sentimiento de pérdida de tiempo, no encontrarse a gusto, tener ganas de trabajar y de obtener bienes materiales o de hacer otras cosas:

[...] yo realmente, lo dejé en tercero de la ESO. Y por varios motivos diferentes: no me gustaba mucho la manera de trabajar en el instituto, con el conjunto de las personas [...] bueno, de hecho quería empezar a trabajar, conseguir ciertas cosas para mí, una moto. Y no me gustaba la forma de trabajo de allí (Francisco, 17)2.

b) Desánimo por los malos resultados académicos, conjugado con el deseo de demostrar que se puede tener éxito en otro contexto: "Te vas desmotivando porque en un examen no sacas la buena nota que esperabas... y ya no estudias tanto... y al final lo dejas correr" (Ana, 20).

c) La imposibilidad de aprobar y pasar de curso, debido a la percepción de un elevado grado de dificultad y el sentimiento de agobio: "[...] nos enviaban por ejemplo de cada materia como trece ejercicios y se me acumulaban y acumulaban y encima te mandaban mucho para estudiar, muchos exámenes pues yo ya me harté y dejé de estudiar" (Pedro, 17).

d) La falta de motivación por el estudio o el rechazo hacia lo escolar: "Acabé cuarto, pero suspendí y podía repetir y sacarme el graduado, pero pasé de aquella escuela, que es una mierda" (Gabriel, 18).

En otros casos, la decisión de abandonar la ESO antes de obtener la titulación de GESO es tomada por la familia o el centro educativo, de forma más o menos pactada con el chico o la chica. Las familias que toman la iniciativa generalmente han encontrado un empleo para su hijo o hija, con frecuencia en la empresa familiar. La decisión del centro puede hacerse operativa por medio de la expulsión del mismo, pero en mayor

2 En todos los casos el nombre es ficticio y el número que aparece junto a este indica la edad en el momento de la entrevista. 
medida, los centros orientan al alumnado hacia Programas de Garantía Social (PGS) o hacia la Educación Secundaria para Personas Adultas (ESPA) ${ }^{3}$.

Una abundante oferta de empleos no cualificados para jóvenes, así como la valoración social del consumo y de lo inmediato, aparecen como factores facilitadores del abandono

La facilidad para encontrar empleo no cualificado es uno de los elementos facilitadores del AEP. Las características del mercado de trabajo de Baleares, donde el turismo y sus sectores asociados de servicios y construcción son los motores de la economía, facilita una salida rápida y "digna" para los jóvenes que por diversas razones no se encuentran a gusto o no hallan sentido a su permanencia en el sistema educativo más allá de la edad de la escolaridad obligatoria. También da la posibilidad de "descansar" del instituto con la idea de volver. Además es un instrumento para los padres que apoyan la experiencia laboral de los hijos para que puedan valorar con una perspectiva más amplia su participación en la formación.

La facilidad para encontrar empleo y los valores asociados con el consumo como facilitadores del abandono quedan documentados en las narraciones. Estas son algunas de las más significativas: "Aparte, yo cuando, antes de... dejarlo, yo ya me había encontrado trabajo iahá! O sea, yo me fui ya teniendo trabajo" (Raquel, 20). "[...] a mí me gusta mucho el dinero, ¿sabes? A mí el dinero... bufff... y también el trabajo, comprar y tener un coche... iMe gusta tener dinero!" (Federico, 20).

No obstante, el análisis de los itinerarios seguidos después de abandonar la escuela muestra que no todos optan por un empleo; también son habituales aquellos en los que la formación es la actividad principal (sobre todo en ESPA o PGS) y, en menor grado, los que combinan trabajo y estudio. Asimismo, hay casos de jóvenes que se quedan en casa sin hacer nada o para realizar actividades diversas (cuidados familiares, trabajo en el entorno familiar, etc.) sin que alguna de ellas predomine con claridad.

Un proceso de desvinculación en lo académico y en lo social que se inicia en distintos momentos, pero que aparece muy ligado al paso de la primaria a la secundaria

El proceso temporal de desvinculación de la escuela puede contemplarse desde la perspectiva académica y social y en relación con las dos etapas de la educación obligatoria.

Desde la perspectiva académica se observan dos grupos: el primero se caracteriza por buenos resultados

3 La Ley Orgánica de Educación de 2006 sustituyó los PGS por los Programas de Cualificación Profesional Inicial (PCPI). No obstante, en el período de las biografías de los jóvenes estudiados todavía no se había hecho tal modificación. en la educación primaria y por la aparición de las primeras dificultades en la ESO. En el segundo, los resultados académicos son insuficientes en la educación primaria (en algunos casos, las dificultades se sitúan en quinto o sexto de primaria y en otros, al principio de la etapa) y se agravan las dificultades en la ESO.

En la mayoría de los casos hay referencias explícitas a una transición difícil entre la educación primaria y la educación secundaria, como determinante de los bajos resultados académicos.

Desde la perspectiva social, los recuerdos y sentimientos asociados con la escuela son, en la mayoría de casos, ambivalentes: se expresan, de forma simultánea, recuerdos y sentimientos tanto positivos como negativos. La situación más común es la que contrapone el paso por la educación primaria (positivo) al de la secundaria (negativo). En estas narraciones se observa la importancia de la dimensión social; así como la relación entre los resultados académicos, los sentimientos hacia la escuela y el "gusto" por esta.

A Marisa (18) le gustó mucho ir a la escuela primaria. Tiene muy buenos recuerdos de sus compañeros, compañeras y del profesorado. En el IES se sintió más sola y menos reforzada: "[...] en el instituto haces lo que quieres... encuentro que te dejan demasiado... iHacer lo que quiera!... allí no están pendientes de ti... si suspendes, has suspendido y si apruebas, has aprobado".

La problemática asociada con la transición entre la etapa de primaria y la de secundaria está estrechamente relacionada con los cambios propios de la adolescencia. En esta línea, Jaime (18) apunta:

[...] han sido unos años que... he pasado de todo y... pero bueno... era... ibuf! Se me hacía pesado, el tener que ir a estudiar... no sé, no te lo tomas en serio, no ves lo que puede pasar en un futuro, no. Solo miras en el presente y no te apetece estudiar, no te apetece y pasas de todo.

También encontramos casos en los que la escuela se liga solo a recuerdos y sentimientos negativos. En ellos predomina el sufrimiento y no hay ningún tipo de identificación con los aspectos académicos o sociales de la escuela. El caso más extremo es el de Gabriel (18), que califica el centro al que iba como "una escuela horrorosa, hablando claro". El hecho de asociar solo recuerdos positivos coincide con los dos casos en los que hubo buen rendimiento académico y el abandono se produjo debido a una situación traumática.

\section{La espiral de desvinculación es tolerada por las escuelas y las familias}

Son los chicos y las chicas quienes, mediante comportamientos que oscilan entre la pasividad más 
absoluta a menudo con elevados índices de absentismo y la algarabía en clase, van avanzando en un proceso que les aleja cada vez más de la cultura y las exigencias de la escuela. En este camino, parece como si los adultos (padres y profesores) y las instituciones (sobre todo la familia y la escuela) se sintieran impotentes y hubieran renunciado a sus obligaciones.

Absentismo, mal comportamiento y pasividad son los tres síntomas más claros que manifiestan los participantes en el estudio durante el proceso de alejamiento progresivo de la escuela que desemboca en el abandono temprano de los estudios. El grupo de iguales desempeña un papel central en estas conductas. También se destacan el ambiente propicio al absentismo y la permisividad en relación con el mismo por parte de los centros.

Aunque el absentismo es un elemento central en el proceso de alejamiento de la escuela, surgen situaciones en las que hay una asistencia regular a clase. Aquí el proceso de alejamiento se evidencia en una presencia puramente física y una pasividad total frente al aprendizaje o comportamientos disruptivos. En todos los casos se crea un círculo vicioso en el que se acumulan las dificultades y el desánimo y se tienen comportamientos cada vez más alejados de los requeridos para el éxito académico. El caso de Ignacio (20) ejemplifica este tipo de situaciones.

[...] era una persona que, que a lo mejor... no lo entendía, o que me costaba más entenderlo y ya no me esforzaba, y ya... volver a preguntar o tal y ya no lo preguntaba. Y ya, pues, perdía y perdía y perdía. Y ya llega un año que... paso [...]. Yo he ido a clase, he asistido a clase. En todos los años que he estado allí...en el instituto... yo expulsado no he estado nunca; no era uno de estos conflictivos, no daba problemas. Pero ir a mi casa y ponerme a estudiar y repasar, no, no. A lo mejor un día me ponía a estudiar pero me costaba bastante y me ponía... no lo entendía.

En cuanto a las familias, se resalta la falta de control derivada de la suplencia temporal de los padres por los abuelos, las dinámicas familiares difíciles, los horarios de trabajo familiares y la dedicación al cuidado de algún miembro de la familia enfermo.

[...] nunca he tenido ningún horario ni ningún control... después de salir de casa de mi abuela... nunca tuve ningún control de nada... tuve libertad absoluta. Pero no porque mi madre pasara de mí, sino porque ella no estaba (Ana, 20).

\section{Discusión y conclusiones}

El estudio de los procesos de abandono escolar y de retorno a la educación por medio de las historias de vida de los jóvenes que están cursando la ESPA nos ofrece una visión privilegiada y oportuna de las razones y de los procesos que conducen al abandono escolar antes de obtener el título de GESO.

El estudio apoya los resultados de las investigaciones precedentes respecto a las principales características del abandono escolar y, a la vez, aporta elementos novedosos respecto al discurso dominante sobre sus causas en España y documenta los procesos de abandono antes de obtener el título de GESO.

En cuanto a las características del abandono escolar, los resultados avalan una multiplicidad de factores que influyen en el mismo y que incluyen aspectos en el ámbito micro, meso y macrosocial (Blaya, 2010; Consejo 
Económico y Social, 2011; Dale, 2010; Desmarais, 2012; Rumberger, 2004; Salvà-Mut \& Sureda-Negre, 2012), que se interrelacionan de distintas formas y con distintas intensidades. El carácter de proceso se refiere a que el abandono escolar no es un hecho puntual y aislado, sino que es el resultado del alejamiento progresivo de la escuela. Este carácter de proceso, junto con la multidimensionalidad de sus causas, requiere de una aproximación holística, lo que hace pertinente la perspectiva biográfica.

Los resultados de esta investigación nos permiten dialogar con el discurso dominante a partir de la visión de los y las jóvenes. Se destaca la deconstrucción del discurso sobre el empleo como "atracción fatal" que conduce al abandono, argumento central en buena parte de los análisis sobre abandono escolar temprano en España y, de manera muy especial, en las comunidades autónomas con un peso importante del turismo y sectores asociados. Este discurso es sustituido por una visión más amplia en la que el elemento central es la interrelación con otros factores, entre los que sobresalen la experiencia escolar (malos resultados académicos asociados con sentimientos de pérdida de tiempo, no encontrarse a gusto, desánimo, deseo de demostrar que se puede tener éxito en otro contexto, falta de motivación, rechazo hacia lo escolar) y los valores sociales dominantes. Las situaciones y los sentimientos que se producen a partir de estas interacciones hacen que, en un momento determinado de la biografía del joven, el empleo sea la opción que le ofrece un mejor balance entre costes y beneficios. También se observan situaciones en las que los y las jóvenes intentan formarse por otras vías, frente a las dificultades de continuar cursando la ESO.

El análisis del proceso de abandono contribuye a profundizar en las interacciones entre la vinculación académica y social con la escuela y en las dificultades específicas de la transición entre la educación primaria y la secundaria. Esta transición, cuyos riesgos frente a la desvinculación de la escuela se documentan en la literatura sobre el tema (Feyfant, 2012; MenaMartínez, Fernández-Enguita \& Riviere-Gómez, 2010; Millet \& Thin, 2005; Morris \& Pullen, 2007), no es objeto de políticas o de prácticas educativas que permitan su prevención.

Las narraciones de los y las jóvenes revelan la influencia del grupo de iguales y las dificultades de los adultos para acompañarles y orientarles en este momento complicado de su biografía. El período del desarrollo que viven y el cambio que supone el paso a la educación secundaria requieren un mayor y más complejo apoyo que etapas anteriores. Por tanto, es imprescindible que los adultos retomemos nuestro papel educador.

En cuanto al proceso de alejamiento progresivo de la escuela, como se refleja en lo que hemos denominado "espiral de desvinculación", hay una enorme tolerancia por parte de los centros educativos y de las familias, reflejo de una tolerancia social más amplia, que es una de las claves para comprender, por una parte, las elevadas tasas de abandono escolar que se producen en general en España y en particular en Baleares y, por otra parte, la debilidad de las políticas y prácticas educativas dirigidas a su disminución.

Los resultados de la investigación sugieren la necesidad de mejorar la vinculación académica (prevención del fracaso escolar) y social con la escuela (clima escolar, actitudes y formación del profesorado, pedagogía centrada en el alumnado) y el establecimiento de políticas que promuevan la compatibilidad entre la formación y el empleo, con medidas claras de apoyo a los y las jóvenes con rendimientos académicos inferiores y/o situaciones de necesidad económica (garantía juvenil, formación dual, ayudas específicas). 
La riqueza de las reflexiones de estos y estas jóvenes (todavía sin título de GESO en el momento de realizar la entrevista) muestra con claridad la relevancia de considerar la voz del alumnado en la investigación educativa. El tipo de resultados que se deriva de esta perspectiva aporta una visión diferente y complementaria a la de otros actores y facilita la transferencia de los resultados de la investigación a las políticas y prácticas educativas.

\section{Sobre los autores}

Francesca Salvà-Mut es doctora en Ciencias de la Educación, de la Universidad de las Islas Baleares (España). Profesora titular adscrita al Departamento de Pedagogía Aplicada y Psicología de la Educación, de la Universidad de las Islas Baleares. Líneas de investigación: abandono educativo temprano, itinerarios formativos y laborales, educación de segunda oportunidad.

Miquel F. Oliver-Trobat es doctor en Pedagogía, de la Universidad de las Islas Baleares (España). Profesor titular adscrito al Departamento de Pedagogía Aplicada y Psicología de la Educación, de la Universidad de las Islas Baleares. Líneas de investigación: abandono educativo temprano, trayectorias de la educación al empleo, educación para la sostenibilidad.

Rubén Comas-Forgas es doctor con Mención Europea en Ciencias de la Educación, de la Universidad de las Islas Baleares (España). Profesor contratado adscrito al Departamento de Pedagogía Aplicada y Psicología de la Educación, de la Universidad de las Islas Baleares. Líneas de investigación: integridad académica, usos sociales y educativos de las TIC.

\section{Agradecimientos}

Los autores forman parte del grupo de investigación Educación y Ciudadanía, de la Universidad de las Islas Baleares, que cuenta con la consideración de Grupo Competitivo y el patrocinio por parte de la Comunidad Autónoma de las Islas Baleares, por medio de la Direcció General de Recerca, Desenvolupament Tecnològic i Innovació de la Conselleria d’Innovació, Interior i Justícia y la cofinanciación de fondos europeos Feder.

La investigación que se presenta ha contado con el apoyo económico de la Dirección General de Formación Profesional y Aprendizaje Permanente de la Consejería de Educación y Cultura del Gobierno Balear.

\section{Referencias}

Adame, T., \& Salvà, F. (2010). Abandono escolar prematuro y transición a la vida activa en una economía turística: el caso de Baleares. Revista de Educación, 35, 185-210.
Bertaux, D. (1997). Les récits de vie perspective ethnosociologique. París: Nathan.

Blaya, C. (2010). Décrochages scolaires. L'école en difficulté. Bruselas: De Boeck.

Casquero, A., \& Navarro, M. (2010). Determinantes del abandono escolar temprano en España, un análisis por género. Revista de Educación, núm. extraordinario 1, 191-213.

Coleman, J. S. (1988). Social Capital in the Creation of Human Capital. The American Journal of Sociology, 94, 95-120.

Consejo de la Unión Europea. (2009). Conclusiones del Consejo de 12 de mayo de 2009 sobre un marco estratégico para la cooperación europea en el ámbito de la educación y la formación ("ET 2020"). Diario Oficial de la Unión Europea, 119, 2-10. Recuperado de http://eur-lex.europa.eu/ LexUriServ/LexUriServ.do?uri=OJ:C:2009:119:00 02:0010:es:PDF

Consejo Económico y Social. (2011). Fracaso y abandono escolar temprano en España. Cauces, 16, 44-81.

Consejo Económico y Social. (2009). Sistema educativo y capital humano. Informe 01/2009. Madrid: Consejo Económico y Social.

Dale, R. (2010). Early School Leaving. Lessons from Research for Policy Makers. Recuperado de http://www.spd.dcu.ie/site/edc/documents/nesse2010early-school-leaving-report.pdf

Desmarais, D. (2009). El enfoque biográfico. Cuestiones Pedagógicas, 20, 27-54. Recuperado de http://institucional.us.es/revistas/cuestiones/20/ art_02.pdf

Desmarais, D. (2012). Contrer le décrochage scolaire par l'accompagnement éducatif. Une étude sur la contribution des organismes communautaires. Québec: Presses de I'Université du Québec.

Eckert, H. (2006). Entre el fracaso escolar y las dificultades de inserción profesional: la vulnerabilidad de los jóvenes sin formación en el inicio de la sociedad del conocimiento. Revista de Educación, 341, 35-56. Recuperado de http://www.revistaeducacion.mec.es/re341/re341_02.pdf

Fernández Enguita, M., Mena, L., \& Riviere, J. (2010). Fracàs i abandonament escolar a Espanya. Barcelona: Fundació La Caixa.

Fernández, E., Muñoz, R., Braña, F., \& Antón, J. (2010). Algunas apreciaciones aritméticas sobre el fracaso y el abandono escolar en España. Revista de Educación, núm. extraordinario 1, 303-324.

Ferraroti, F. (2011). Las historias de vida como método. Acta Sociológica, 56, 95-119.

Feyfant, A. (2012). Enseignement primaire: les élèves à risque (de décrochage). Dossier d'actualité Veille et Analyses, 80, 1-18. 
García, M., Casal, J., Merino, R., \& Sánchez, A. (2013). Itinerarios de abandono escolar y transición tras la ESO. Revista de Educación, 361, 65-94.

González, M. T. (2006). Absentismo y abandono escolar: una situación singular de la exclusión educativa. Revista Electrónica Iberoamericana sobre Calidad, Eficacia y Cambio en Educación, 4 (1), 1-15.

Häfeli, K., \& Schellenberg, C. (2009). Facteurs de réussite dans la formation professionnelle de jeunes à risque. Berne: Conférence suisse des directeurs cantonaux de l'instruction publique (CDIP).

Hernández, F., Sancho, J. M., \& Rivas, J. I. (2011). Historias de vida en educación. Biografías en contexto. Recuperado de http://hdl.handle. net/2445/15323

Johnson, A. S. (2007). An Ethics of Access: Using Life History to Trace Preservice Teachers' Initial Viewpoints on Teaching for Equity. Journal of Teacher Education, 58, 299-314.

Lamamra, N., \& Masdonati, J. (2009). Arrêter une formation professionnelle. Mots et maux d'apprenti.e.s. Lausana: Antipodes.

Lee, V.E., \& Burkam, D.T. (2003). Dropping Out of High School: The Role of School Organization and Structure. American Educational Research Journal, 40 (2), 353-393.

Martínez, R.A., \& Álvarez, A. (2005). Fracaso y abandono escolar en la educación secundaria obligatoria: implicación de la familia y los centros escolares. Aula Abierta, 85, 127-146. Recuperado de http://redined.mecd.gob.es/xmlui/handle/11162/4373

Mena-Martínez, L., Fernández-Enguita, M., \& RiviéreGómez, J. (2010). Desenganchados de la educación: procesos, experiencias, motivaciones y estrategias del abandono y del fracaso escolar. Revista de Educación, núm. extraordinario, $119-146$

Merete, B. A. (2006). Young People's Subjective Understandings of Early School Leaving in Rural Areas. Recuperado de http://eprints.utas.edu. au/905/

Millet, D., \& Thin, D. (2005). Ruptures scolaires. L'école à l'épreuve de la question sociale. París: PUF.

Ministerio de Educación, Cultura y Deporte. (2013). Explotación de las variables educativas de la encuesta de población activa: nivel de formación y formación permanente. Recuperado de https:// www.educacion.gob.es/horizontales/estadisticas/mercado-laboral/Explotaci-n-de-las-variables.html
Mora, A. J. (2010). Determinantes del abandono escolar en Cataluña, más allá del nivel socio-económico de las familias. Revista de Educación, núm. extraordinario 1, 171-190.

Morris, M., \& Pullen, C. (2007). Disengagement and Re-Engagement of Young People in Learning at Key Stage 3. Totnes: Research in Practice.

Pujadas, J. J. (1992). El método biográfico: el uso de las historias de vida en ciencias sociales. Madrid: CIS.

Rioux, C. (1998). L'horizon de référence et l'accrochage scolaire. Recuperado de http://amicus.collectionscanada.gc.ca/aaweb-bin/aamain/itemdisp? sessionKey $=999999999 \_142 \mathrm{yl}=1 \mathrm{yd}=2 \mathrm{yv}=0 \mathrm{ylvl}$ $=1$ yitm $=38280776$

Rivas, J. I., Hernández, F., Sancho, J. M., \& Núñez, C. (coord.) (2012). Historias de vida en educación: Sujeto, diálogo, experiencia. Recuperado de http://hdl.handle.net/2445/32345

Roksa, J., \& Velez, M. (2010). When Studying Schooling is not enough: Incorporating Employment in Models of Educational Transitions. Research in Social Stratification and Mobility, 28, 5-21.

Rumberger, R. W. (2004). Why Students Drop Out of School. En G. Orfield (ed.). Dropouts in America: Confronting the Graduation Rate Crisis. Cambridge: Harvard Education Publishing Group.

Salvà-Mut, F., \& Sureda-Negre, J. (2012). L'utilisation des histoires de vie dans la recherche et I'innovation de l'école de la deuxième chance. En D. Desmarais, I. Fortier \& J. Rhéaume (eds.). Transformations de la modernité et pratiques (auto)biographiques. Québec: Presses de I’Université du Québec.

Savoie-Zajc, L. (2011). La recherche qualitative/interprétative en éducation. En T. Karsenti \& L. Savoie-Zajc (eds.). La recherche en éducation. Étapes et approches. Quebec: Éditions du Renouveau Pédagogique Inc.

Simard, J. (1997). Étude descriptive des facteurs scolaires influençant l'abandon des études chez les élèves du secondaire. Recuperado de http:// constellation.uqac.ca/1095/1/10983642.pdf

Smale, W. T. (2001). Understanding the Issue of Dropouts: A Young Offender Perspective. Recuperado de http://amicus.collectionscanada.gc.ca/ aaweb-bin/aamain/itemdisp? sessionKey $=99999$ 9999_142yl=1yd=2yv=0ylvl=1yitm $=25403399$

Vaillancourt, C. (1998). Le décrochage scolaire: une approche communicationnelle. Recuperado de http://www.collectionscanada.gc.ca/obj/s4/f2/ dsk1/tape8/PQDD_0019/MQ46504.pdf 